\title{
INTEGRAÇÃO ELÉTRICA AO SISTEMA DE AUTOMAÇÃO DE PROCESSOS UTILIZANDO IEC 61850*
}

Alcio Heitor Somensari ${ }^{1}$ Fernando Keisuke Tani ${ }^{2}$

\section{Resumo}

O artigo apresenta um estudo de como um sistema de automação elétrica integrado deve fornecer uma completa visão do sistema elétrico, permitindo que as equipes de operação e manutenção tomem decisões precisas rapidamente após uma falha elétrica. As indústrias esperam que suas subestações estejam sempre disponíveis e ligadas, evitando paradas indesejadas. Para isso, precisam de soluções que recuperem o fornecimento de energia de forma rápida e segura, a um custo mínimo. Proporcionar um ambiente aprimorado na sala de controle para uma rápida solução de problemas e conectar subestações de locais hostis e distantes reduzem não apenas tempo de manutenção, mas também aumentam a segurança das equipes. $A$ norma IEC 61850 nos permite entregar uma solução para um sistema mais intuitivo com acesso rápido a informações que melhoram a supervisão da planta na sala de controle.

Palavras-chave: Integração elétrica; Automação elétrica; IEC 61850.

\section{ELECTRICAL INTEGRATION TO THE PROCESS AUTOMATION SYSTEM USING IEC 61850}

\section{Abstract}

The article presents a study of how an integrated electrical automation system has to allow a full insight into the electrical system enabling operators and maintenance teams to take faster and more accurate decisions after an electrical fault. Industrial companies expect the substations to be always available and powered on avoiding undesired stoppages. For that, an industry needs solutions that recover the energy supply quickly and safely at minimum cost. Providing an enhanced environment at the control room for faster troubleshooting and connecting substations from distant and harsh places saves not only maintenance time but also improves safety for staff. The IEC 61850 standard allow us to deliver a solution for a more intuitive system with quick information access that improves the plant supervision at the control room.

Keywords: Electrical integration; Power automation; IEC 61850.

1 Engenheiro eletricista, engenheiro de projetos e desenvolvimento - automação elétrica, automação de processos industriais, ABB, Osasco, SP, Brasil.

2 Engenheiro eletricista, mestre em sistemas digitais, coordenador de engenharia - automação elétrica, automação de processos industriais, ABB, Osasco, SP, Brasil. 


\section{INTRODUÇÃO}

As indústrias esperam que suas subestações estejam sempre disponíveis e ligadas, evitando paradas indesejadas. Para isso, precisam de soluções que recuperem o fornecimento de energia de forma rápida e segura, a um custo mínimo. Uma vez que ambientes de mineração possuem subestações distantes umas das outras, para as operações de manutenção em caso de falhas elétricas, deve-se proporcionar um ambiente aprimorado na sala de controle para uma rápida solução de problemas remotamente evitando grandes perdas de tempo no deslocamento destas equipes até a subestação. Além disso, conectar as subestações de locais hostis e distantes reduzem não apenas tempo de manutenção, mas também aumentam a segurança das pessoas.

Dentro deste cenário, deve-se estar disponível aos times que operam a planta, um sistema supervisório na sala de controle que permita ter uma visão aprimorada de todas as subestações para uma imediata compreensão do problema, assim como permitir que operações sejam realizadas para uma primeira tomada rápida de ação. Para isso, torna-se necessário um protocolo de comunicação que permita uma gama de funcionalidades para as tarefas de supervisão, operação, medição e manutenção. Desde 2004 a norma IEC 61850 nos permite entregar uma solução para um sistema mais intuitivo com acesso rápido a informações que melhoram a supervisão da planta na sala de controle permitindo a execução de tarefas antes apenas realizada localmente na subestação.

\subsection{Norma IEC 61850}

IEC 61850 é uma norma global para o projeto de automação de subestação elétrica e sistemas de distribuição de energia. É uma norma definida pelo trabalho conjunto de ambas organizações ANSI (América do Norte) e IEC (Europa) juntamente com diversos fabricantes no mercado de automação de subestações.

A motivação para criação da norma IEC 61850 surgiu da necessidade de se estabelecer uma padronização na comunicação entre os dispositivos de controle e proteção dos equipamentos (disjuntor, transformador, etc.) das subestações de energia elétrica, uma vez que era comum aos diversos fabricantes desenvolver seu próprio protocolo tornando obrigatória a utilização de equipamentos conversores de protocolo para tradução e centralização dessas informações dificultando, assim, a execução de projetos de automação de subestações e a manutenção das redes de comunicação.

Lançada inicialmente em 2004, IEC 61850 define modelos de dados a certos protocolos que utilizam redes TCP/IP ou redes locais de subestação através de equipamentos Ethernet utilizando diversos dispositivos eletrônicos inteligentes (IED - intelligent electronic device) para desempenhar as funções exigidas como proteção, controle e monitoramento local e remoto.

A norma IEC 61850 tem como base três metas: Interoperabilidade, liberdade de configuração e estabilidade a longo prazo.

A interoperabilidade é a capacidade dos IEDs de diferentes fabricantes de se comunicarem entre si. A liberdade de configuração é a capacidade de cada fabricante em utilizar métodos e filosofias na programação interna dos IEDs, contanto que os dados transmitidos entre os equipamentos obedeçam a meta de interoperabilidade. A estabilidade a longo prazo refere-se à capacidade dos 
protocolos de comunicação em permanecerem atualizados frente a avanços tecnológicos.

A abordagem da norma IEC 61850 é combinar os pontos fortes dos três seguintes métodos: Decomposição funcional, modelagem de fluxo de dados, modelagem de informação.

Decomposição funcional é utilizada para compreender a relação lógica entre os componentes de uma função distribuída, e é apresentado em termos de nós lógicos que descrevem as funções, subfunções e interfaces funcionais. O fluxo de dados é utilizado para compreender as interfaces de comunicação que deve suportar o intercâmbio de informações entre os componentes funcionais distribuídos e os requisitos de performance funcional. A modelagem de informações é utilizada para definir a sintaxe abstrata e semântica das informações trocadas, e é apresentado em termos de classes de objetos de dados e tipos, atributos, métodos de objetos abstratos (serviços), e seus relacionamentos [1].

\subsection{Tendências Globais e os Principais Desafios em Mineração}

Em um cenário cada vez mais competitivo, a busca por métodos de operação cada vez mais produtivos surgem a fim de se alcançar a melhor taxa de produção e consumo de energia. Como consequência, todo novo método aplicado a um processo industrial acarreta em investimentos de bens de capital para novas instalações (projetos greenfield) e gastos operacionais (característico em projetos brownfield). Além disso, uma das grandes questões que entornam o ambiente produtivo de mineração é a segurança das equipes de manutenção e operação da planta. Tornar o ambiente de supervisão e controle da planta mais eficaz e seguro dispondo de tecnologia para reduzir os investimentos são os principais desafios a destinar-se o foco.

\subsubsection{Reduzir o investimento de bens de capital e gastos operacionais}

Quando nos referimos a redução de investimentos de bem de capital, CAPEX (Capital Expenditure), que é um investimento característico de plantas novas (greenfields). A principal preocupação na redução de custos é no alto valor de implementação dos cubículos de eletrificação e no cabeamento utilizado para interligá-los.

Visto que por muitas vezes estas interligações entre os cubículos são padronizadas em típicos de projeto elétrico, pode-se ao invés de utilizarmos cabos fiados para a troca de informações, dispormos de um protocolo de comunicação confiável e que seja rápido o suficiente que fornece a mesma solução em nível de funcionalidade e confiabilidade de sistema.

Para esta solução, a norma IEC 61850 padroniza o protocolo de comunicação horizontal designado GOOSE [1] (Generic Object Oriented Substation Event). Através do protocolo GOOSE informações podem ser trocadas entre os IEDs dos cubículos evitando, assim, que diversos cabos e fios sejam passados entre os cubículos, mas sim, apenas uma fibra óptica de comunicação para estabelecer esta comunicação via rede Ethernet.

É possível identificar padrão de interligação entre os cubículos, e geralmente este padrão repete-se várias vezes dentro de uma subestação (ou em diversas subestações de uma planta). Isto permite trabalhar, também, com típicos de programação de IEDs tornando o comissionamento mais rápido, o que reduz custos e melhorando a segurança das equipes de projeto. 
Como o cabeamento e fiação é reduzido, pode-se reduzir consideravelmente o tamanho das salas elétricas (E-houses) reduzindo assim os custos de infraestrutura e refrigeração dos equipamentos elétricos presentes no interior destas salas.

Além de toda a redução de investimentos em CAPEX, tem-se a preocupação de redução dos gastos operacionais denominado OPEX (Operational Expenditure). Estes são facilmente reduzidos através de uma engenharia padronizada que permite menos horas de desenvolvimento de projeto, e um comissionamento mais rápido devido a pré-programação dos típicos de IED.

\subsubsection{Aumentar a disponibilidade do fornecimento de energia para o processo}

Dentre as principais questões abordadas nas paradas indesejadas em uma planta de mineração está uma que pode-se classificar como a principal na improdutividade: Falta de disponibilidade de energia elétrica.

Espera-se na ocasião de uma de uma falha elétrica que as subestações estejam em $100 \%$ de sua totalidade disponíveis ao fornecimento de energia aos ativos da planta. Isto é alcançado através de uma rápida solução do problema elétrico.

Para que as equipes de manutenção e operação consigam rapidamente disponibilizar a subestação deve-se dispor de um sistema que permita solução de problemas remotamente, em que torne-se possível visualizar todas as informações de proteções, dados de oscilografia, diretamente da sala de controle através de um sistema que seja intuitivo operacionalmente. Através da implementação de IEC 61850 na automação elétrica o número de diferentes tecnologias é reduzido tornando menos complexo a tarefa de análise e manutenção dos dispositivos de proteção.

\subsubsection{Segurança para as equipes}

Com a implementação da norma IEC 61850, torna-se possível configurar e programar os relés de proteção de controle remotamente através da sala de controle, mantendo as equipes longe do risco elétrico. Estas operações não são possíveis de serem executadas com os mais diversos protocolos de comunicação existentes e em grande escala, empregados nas subestações (como por exemplo MODBUS, PROFIBUS).

Aliado a facilidade de se acessar e configurar os dispositivos diretamente da sala de controle, um sistema de automação elétrica integrado com IEC 61850 permite conectar as subestações de locais distantes e hostis. Desta forma, diminui-se a exposição das equipes de manutenção dos perigos iminentes de falhas nos equipamentos de subestação como disjuntores, chaves secionadoras, trazendo mais segurança e ergonomia nas atividades de reparo e manutenção.

\section{INTEGRAÇÃO ELÉTRICA AO SISTEMA DE AUTOMAÇÃO DE PROCESSOS}

Tradicionalmente, automação de processos e automação elétrica são implementadas em sistemas distintos dentro de plantas industriais. A automação de processos, feita em grande parte através de sistemas DCS, é responsável pelo controle e monitoração do processo através da interface com a instrumentação e os equipamentos de baixa tensão. A automação elétrica, implementada geralmente por sistemas SCADA, é responsável pela distribuição elétrica na planta e envolve os equipamentos de controle e proteção elétrica das subestações, mas pode também incluir soluções avançadas de controle e gerenciamento de energia. Tipicamente há uma sobreposição dos sistemas nos equipamentos de média tensão, como drives e 
motores, que são controlados pelo processo, mas geralmente considerados como parte do sistema elétrico.

Ter estes dois sistemas distintos dentro de uma mesma planta representa diversas desvantagens do ponto de vista técnico e econômico. A falta de padronização entre os diversos protocolos disponíveis para o sistema elétrico, a dificuldade de se transferir informação entre os dois sistemas para um melhor gerenciamento da planta, e a necessidade de dar manutenção em diferentes produtos e tecnologias são apenas algumas delas.

Reunir os dois sistemas em um único, que pode-se denominar de integração elétrica (Figura 1), apresenta-se então como solução desejada e uma evolução lógica. A integração elétrica fornece um ambiente unificado na sala de controle, que melhora a produtividade, aumenta a segurança e reduz os custos. A próxima geração de operadores não irá mais tomar decisões apenas por meio do monitoramento de tensões e temperaturas, mas sim também em função de reais e centavos. Integrar ambas, automação de processo e elétrica, permite aos operadores uma visão completa de toda a planta. Com a norma IEC 61850 um sistema de automação pode ser desenvolvido para lidar com todas as áreas existentes em uma planta de um único lugar.

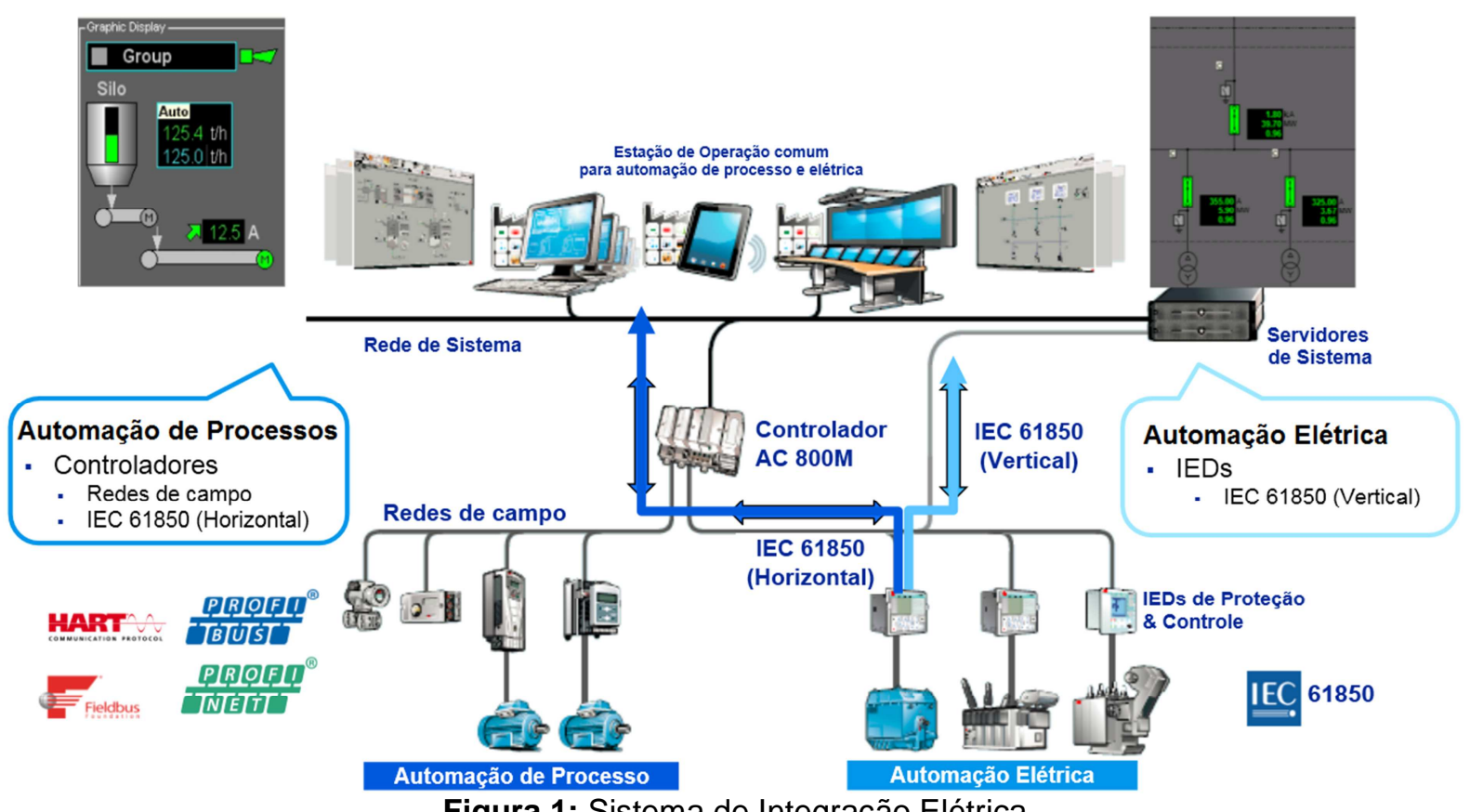

Figura 1: Sistema de Integração Elétrica

\subsection{Benefícios de um Sistema de Integração Elétrica}

As indústrias querem maximizar sua produção mantendo seu processo em operação sem paradas indesejadas. Solucionar problemas de forma rápida e eficiente permite aos operadores e equipes de manutenção reduzir as intervenções e os tempos de parada. Os principais benefícios da integração elétrica incluem:

Sistema único - Promove a colaboração e a eficácia do operador. A troca e cruzamento de dados entre processo e elétrica torna a tomada de decisão mais assertiva e o diagnóstico da causa raiz de problemas mais rápido e eficiente.

Visualização total da planta através de uma única estação de trabalho - Automação de processos e elétrica compartilhando o mesmo ambiente promove uma visão holística da planta tanto para sua operação quanto manutenção. 
Menor custo com peças de reposição, treinamentos e atualizações - Utilizar o mesmo sistema de automação permite otimizar o estoque de peças sobressalentes, reduzir e concentrar a gama de equipamentos e tecnologias empregadas e consequentemente a reduzir a quantidade de treinamentos necessários. Isso facilita a atualização do conhecimento técnico das equipes e também permite que haja um maior aprofundamento deste conhecimento. Além disso, a atualização do sistema de automação em si torna-se mais barato, uma vez que é único.

Estratégia consistente para gerenciamento de ativos - Integração da informação reduz custos através da detecção precoce de problemas nos ativos da planta. $O$ cruzamento de dados permite um diagnóstico mais preciso da saúde dos equipamentos, auxiliando na implementação de uma estratégia consistente de manutenção para evitar quebras e paradas de produção.

Visibilidade aprimorada sobre o consumo de energia - A disponibilidade de uma vasta quantidade de informação de alta qualidade sobre o processo e a elétrica permite a implementação de sistemas de gerenciamento de energia, que monitoram a produtividade versus consumo de energia de diversas partes do processo e auxiliam na otimização da produção com redução de custos.

\subsection{Funcionalidades de um Sistema de Automação Elétrica Eficiente}

Ao integrar os sistemas de automação de processos e elétricas, uma série de funcionalidades são necessárias para atender às necessidades de redução de gastos, segurança para equipes e aumento da disponibilidade de energia em plantas de mineração. Certas funcionalidades são essenciais a fim de se tornar um sistema de integração de fato eficiente e que seja intuitivo e de fácil operação. Dividem-se em 3 grupos: Sistema otimizado, Solução de problemas remotamente e Engenharia otimizada.

\subsubsection{Sistema otimizado}

Um sistema otimizado contribui tanto na fase de implementação, quanto na fase de operação e manutenção do sistema elétrico. Podemos citar:

Automação de processo e elétrica unificadas - Automação de processo e elétrica no mesmo sistema de controle da planta compartilhando da mesma base de dados, ferramentas de engenharia e estações de operação. Um sistema unificado traz melhor padronização para novos funcionários, pois dispõe-se de um único sistema para aprender, além de reduzir custos em treinamentos devido ao conhecimento de um sistema em comum. Por compartilharem a mesma arquitetura, há a otimização de peças de reposição.

Controle de motores de MT com IEC 61850 (sem conversores de protocolo ou gateways) provenientes da Automação de Processo - controladores de Processo podem enviar comandos GOOSE diretamente para os IEDs dos motores de MT: não é necessário fiação ou barramentos seriais entre os sistemas de automação de processo e elétrica Protocolos digitais / Ethernet (Figura 2) 


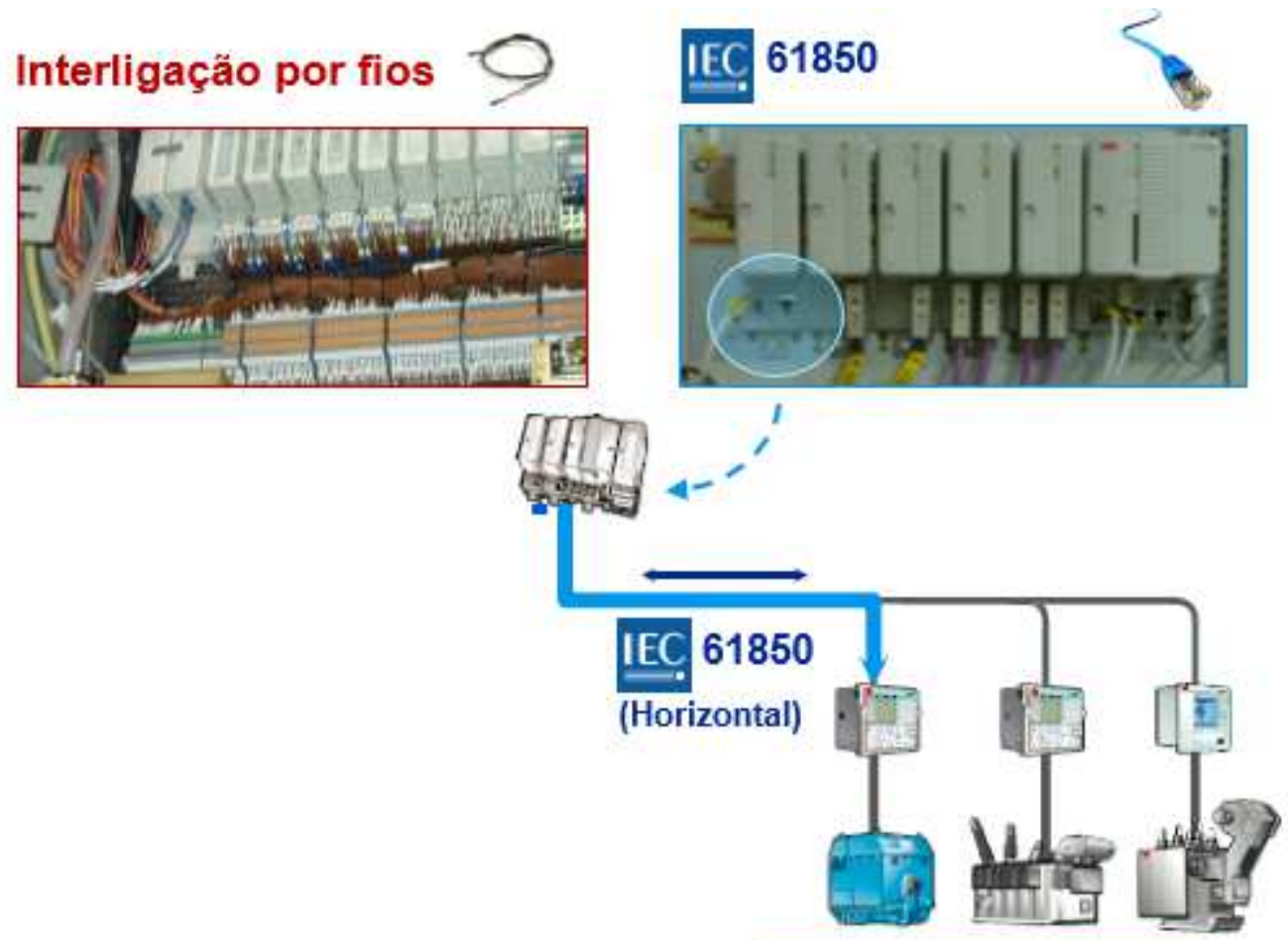

Power Automation

Figura 2: Envio de comando de motores de MV por mensagens GOOSE

Tprna-se possível conectar subestações de locais distantes devido ao uso de protocolos digitais/ Ethernet para comunicação entre os IEDs (IEC 61850) permitindo 0 acesso remoto permite à equipe de manutenção ir até a subestação somente quando necessário.

\subsubsection{Solução de problemas remoto}

Lista de alarmes baseada na sequência de eventos - Compreensão mais fácil da causa/efeito após uma falha elétrica com informação disponível remotamente

Intertravamentos e proteções em faceplates - Mesma informação sobre intertravamentos e proteções localmente e remotamente, de maneira sintetizada, permitindo solucionar problemas mais rapidamente

Acesso a arquivos de registro de distúrbios - Não é necessário ir até a subestação para carregar ou ver os registros, permitindo uma análise mais rápida e segura de falhas

Parametrização e configuração de lógicas do IED - Atalhos para o software de parametrização e configuração de lógicas do IED com apenas um clique, permitindo acesso rápido, economizando tempo da equipe de manutenção

Acesso a documentação online - Diagramas unifilares, diagramas lógicos, manuais técnicos com apenas um clique: Equipe de manutenção vai ao lugar de falha com as informações necessárias em mãos

\subsubsection{Engenharia otimizada}

Com uma engenharia padronizada, pode-se empregar metodologia de engenharia para o desenvolvimento mais rápido de um projeto e garantir o desenvolvimento e comissionamento mais rápidos de um projeto, reduzindo seus riscos e diminuindo tempo de manutenção para projetos de médio a grande porte. 


\section{CONCLUSÃO}

Com o avanço tecnológico e a adoção de novos padrões como o IEC 61850, é possível extrair do sistema de automação elétrico muito mais que simplesmente a supervisão e operação da distribuição elétrica de uma planta. Funcionalidades avançadas como acesso remoto a configuração e parametrização de IEDs, análise de arquivos de oscilografia permitem o diagnóstico e a resolução de problemas de forma mais rápida e eficiente, reduzindo o tempo de parada do processo. A disponibilidade de dados de todo sistema elétrico e o seu controle remoto reduzem a exposição ao risco, aumentando a segurança para as equipes de operação e manutenção.

A integração elétrica, agregando a automação de processo e elétrica em um mesmo sistema, gera sinergias e promove novas funcionalidades que se traduzem em redução de custos e plantas energeticamente mais eficientes. Tais benefícios já são realidade hoje, sendo implementados através de subestações com IEC 61850 e sistemas avançados de automação, como o Sistema 800xA da ABB.

\section{REFERÊNCIAS}

1 IEC/TR 61850-1: Communication networks and systems for power utility automation Part 1: Introduction and overview. Edition 2. 